\title{
Suppressing beam-centroid motion in a long-pulse linear induction accelerator
}

\author{
Carl Ekdahl, ${ }^{1}$ E. O. Abeyta, ${ }^{1}$ R. Archuleta, ${ }^{1}$ H. Bender, ${ }^{2}$ W. Broste, ${ }^{2}$ C. Carlson, ${ }^{2}$ G. Cook, ${ }^{1}$ \\ D. Frayer, ${ }^{2}$ J. Harrison, ${ }^{1}$ T. Hughes, ${ }^{3}$ J. Johnson, ${ }^{1}$ E. Jacquez, ${ }^{1}$ B. Trent McCuistian, ${ }^{1}$ N. Montoya, ${ }^{1}$ \\ S. Nath, ${ }^{1}$ K. Nielsen, ${ }^{1}$ C. Rose, ${ }^{1}$ M. Schulze, ${ }^{1}$ H. V. Smith, ${ }^{1}$ C. Thoma, ${ }^{4}$ and C. Y. Tom ${ }^{2}$ \\ ${ }^{1}$ Los Alamos National Laboratory, Los Alamos, New Mexico 87545, USA \\ ${ }^{2}$ National Security Technologies, Los Alamos, New Mexico 87544, USA \\ ${ }^{3}$ Sandia National Laboratories, Albuquerque, New Mexico 87185, USA \\ ${ }^{4}$ Voss Scientific, Albuquerque, New Mexico 87108, USA \\ (Received 25 July 2011; published 5 December 2011)
}

\begin{abstract}
The second axis of the dual-axis radiography of hydrodynamic testing (DARHT) facility produces up to four radiographs within an interval of $1.6 \mu \mathrm{s}$. It does this by slicing four micropulses out of a $2-\mu \mathrm{s}$ long electron beam pulse and focusing them onto a bremsstrahlung converter target. The 1.8-kA beam pulse is created by a dispenser cathode diode and accelerated to more than $16 \mathrm{MeV}$ by the unique DARHT Axis-II linear induction accelerator (LIA). Beam motion in the accelerator would be a problem for multipulse flash radiography. High-frequency motion, such as from beam-breakup (BBU) instability, would blur the individual spots. Low-frequency motion, such as produced by pulsed-power variation, would produce spot-to-spot differences. In this article, we describe these sources of beam motion, and the measures we have taken to minimize it. Using the methods discussed, we have reduced beam motion at the accelerator exit to less than $2 \%$ of the beam envelope radius for the high-frequency BBU, and less than $1 / 3$ of the envelope radius for the low-frequency sweep.
\end{abstract}

DOI: 10.1103/PhysRevSTAB.14.120401

\section{INTRODUCTION}

The dual-axis radiography for hydrodynamic testing (DARHT) facility produces flash radiographs of highexplosive driven hydrodynamic experiments. Two linear induction electron accelerators (LIAs) make the bremsstrahlung radiographic source spots for orthogonal views of each test. The 2-kA, 20-MeV Axis-I LIA creates a single 60-ns radiography pulse. The Axis-II LIA has a long (1.6- $\mu \mathrm{s}$ ) flattop with energy variation less than $\pm 1.5 \%$. For radiography, it has been operated at more than $16 \mathrm{MeV}$ with beam currents greater than $1.5 \mathrm{kA}$. It creates up to four short radiography pulses by kicking them out of the long flattop. Typical radiography source spots for both axes are $\sim 1$-mm or less FWHM.

The Axis-II LIA uses a hot dispenser cathode to produce the beam, which is initially accelerated in a diode and then by six injector induction cells followed by 68 induction cells in the main accelerator. It uses solenoidal magnetic fields to transport and focus the beam. Each induction cell incorporates a solenoid (for focusing) and a pair of orthogonal dipole coils (for steering). The repetition rate is limited by purging of pulse-power spark gaps to less than one shot per 5 minutes. The Axis-II LIA, the beam it produces, and its solenoidal focusing are further described

Published by the American Physical Society under the terms of the Creative Commons Attribution 3.0 License. Further distribution of this work must maintain attribution to the author(s) and the published article's title, journal citation, and DOI.
PACS numbers: 29.27.Bd, 29.27.Eg, 41.75.Ht, 41.85.Ja

in Refs. [1-3]. After the long pulse exits the accelerator it passes through a kicker, which slices out shorter pulses to be transported to the bremsstrahlung converter target. The remainder of the long pulse is diverted to a beam dump. The kicker and downstream transport (DST) to the bremsstrahlung converter are described in detail in Ref. [4]. The beam simulation codes used to clarify beam physics and design the solenoidal focusing are described in Refs. [5-9].

We have made an effort to understand and reduce beam motion in the Axis-II accelerator, because motion would be a problem for multiple-time radiography. Integration of fast (ns time scale) motion over the multi-ns radiographic pulses would blur and enlarge the source spots. Slower ( $\mu$ s time scale) motion would produce spot-to-spot wander. Such wandering would also distort the spots of the last pulses in the sequence due to asymmetric target erosion by the preceding pulses.

\section{THEORY AND EXPERIMENTAL CONTROL OF BEAM MOTION}

Noninvasive DARHT-II beam diagnostics, such as beam position monitors (BPMs), were used on every shot $[2,3]$. BPMs were located throughout the injector, accelerator, and DST. BPMs separated by $\sim 5 \mathrm{~m}$ throughout the accelerator measure current and position, while the BPMs after the exit had the eight detectors required to also provide unequivocal ellipticity measurements $[2,10]$. Most of the 12 BPMs in the downstream transport had eight detectors for ellipticity measurements because of the quadrupole magnets used for DST transport. Figure 1 shows BPM 


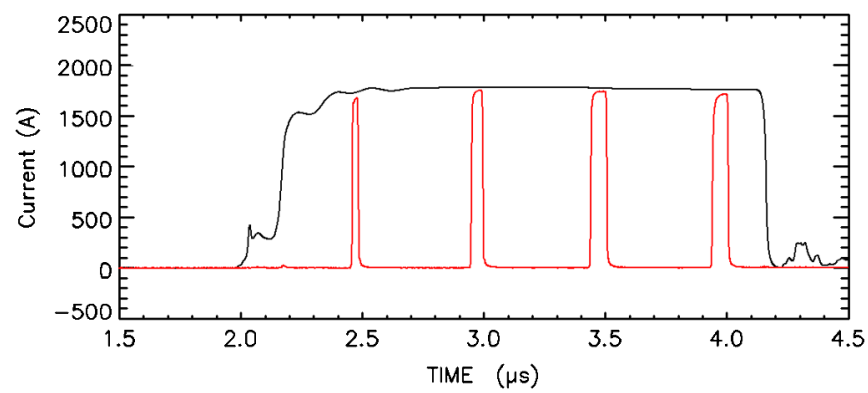

FIG. 1. Overlay of current at accelerator exit and after the kicker, showing the long accelerated-current pulse (black) and four kicked-current pulses (red).

measurements of the long current pulse accelerated by the Axis-II LIA and a sequence of shorter kicked pulses in the DST. Invasive diagnostics were only occasionally used. These included a magnetic spectrometer to measure beam-electron kinetic energy, and time-resolved imaging of the beam current profile using Cerenkov emitters $[1,2,4,11]$. Once the tune of the magnetic focusing and steering has been set, the beam is very reproducible. The day-to-day variation of beam position at the exit is less than $0.5 \mathrm{~mm}$, which is about $10 \%$ of the $\sim 5-\mathrm{mm}$ beam radius predicted by our envelope codes.

\section{A. Suppression of high-frequency motion}

High-frequency beam motion, with period less than the FWHM of the kicked pulse, would increase the radiographic source spot size, by integrating the position over the FWHM. One source of high-frequency motion in our accelerator is the beam-breakup (BBU) instability, which plagues all high-current electron linear accelerators. BBU results from beam coupling of accelerating-cell $\mathrm{TM}_{1 \mathrm{n} 0}$ electromagnetic cavity modes. Since the Axis-II cells have TM mode resonances higher than $100 \mathrm{MHz}$, largeamplitude beam-breakup instability (BBU) would blur the spots of our many-ns radiographic pulses. Therefore, we have taken precautions to suppress this instability both through the design and construction of the cells and through the tuning of the accelerator focusing fields.

For the high-current, strongly focused DARHT-II LIA, the BBU amplitude rapidly grows in time back from the beam head and then saturates due to cavity losses. The saturated amplitude of the beam displacement at a particular location $z$ is

$$
\xi(z)=\xi_{0}\left(\gamma_{0} / \gamma\right)^{1 / 2} \exp \left(\Gamma_{m}\right),
$$

where subscript zero denotes initial conditions, and $\gamma$ is the relativistic mass factor [3,12]. The maximum growth exponent at the location $z$ is

$$
\Gamma_{m}(z)=\frac{I_{b} N_{g} Z_{\perp}}{3 \times 10^{4}}\left\langle\frac{1}{B_{z}}\right\rangle .
$$

Here $I_{b}$ is the beam current in kA, $N_{g}$ is the number of gaps (cells), the cell transverse impedance $Z_{\perp}$ is in $\Omega / \mathrm{m}$, and the $\left\langle 1 / B_{z}\right\rangle$ is the average over $z$ in $\mathrm{kG}^{-1}$, where $B_{z}$ is the solenoidal focusing field. At a particular location in the accelerator, this maximum is reached in the time $\tau=(2 Q / \omega) \Gamma_{m}$ after the arrival of the beam head, where $Q$ is the cavity quality factor and $\omega$ is the mode frequency. This time is less than $25 \mathrm{~ns}$ in the Axis-II LIA.

Based on this theory, BBU can be suppressed by reducing the transverse impedance and increasing the focusing fields. The Axis-II LIA accelerating cells incorporate ferrite tiles to reduce $Z_{\perp}$ by damping the TM modes responsible for the BBU. The other step we have taken to suppress $\mathrm{BBU}$ is to use very strong solenoidal focusing fields. The predicted scaling for $\Gamma_{m}$ was confirmed in earlier experiments [3], so we used this scaling to design a tune with magnetic field strong enough to suppress the BBU to an amplitude $<10 \%$ of beam radius. Figure 2 shows the beam envelope calculated by our envelope code XTR [5] for one of our magnetic field tunes, with the saturated BBU amplitude predicted by this theory overplotted. Calculations of saturated growth for this plot used the maximum measured transverse impedances at the major BBU frequencies bands $[13,14]$ near 150, 250, and $600 \mathrm{MHz}$.

Figure 3 is an example of the beam motion during a 200-ns window near the end of the beam pulse as measured with a BPM at the accelerator exit. The BBU at $\sim 150 \mathrm{MHz}$ is clearly present, but the amplitude is less than 60 microns, which is $<2 \%$ of the $\sim 5$-mm beam radius predicted by envelope code simulations. As described in Ref. [3], we performed extensive BBU experiments with an early 50-cell configuration of the Axis-II LIA to confirm the theoretically predicted scaling of saturated growth of an accelerated beam in the relevant high-current, strongly

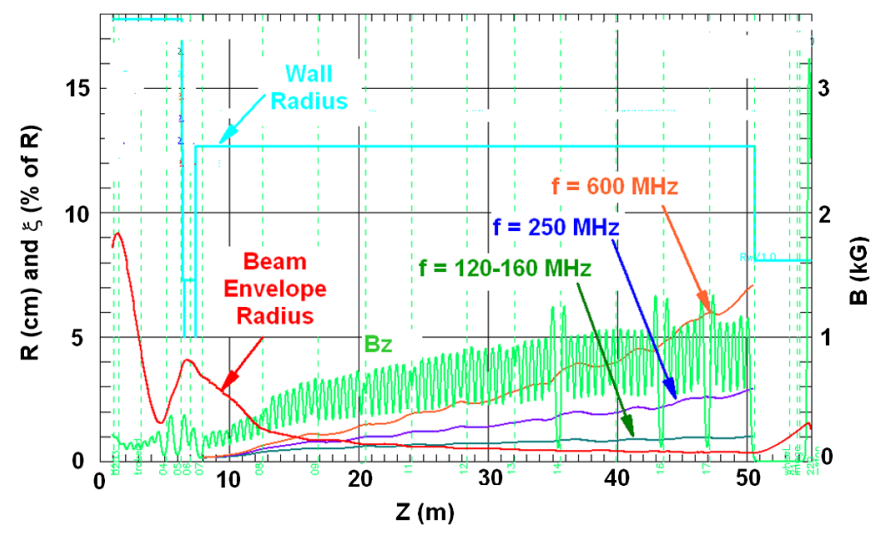

FIG. 2. Beam envelope simulation for a representative tune. On axis magnetic field is plotted in green, with scale at right. Also shown is the saturated growth of the three principal modes of BBU for a 50-micron initial perturbation. Saturated growth of an initial $50-\mu \mathrm{m}$ perturbation based on Eq. (1) is shown as a percentage of beam radius (left scale). The locations of BPMs are shown as vertical green dotted lines. 

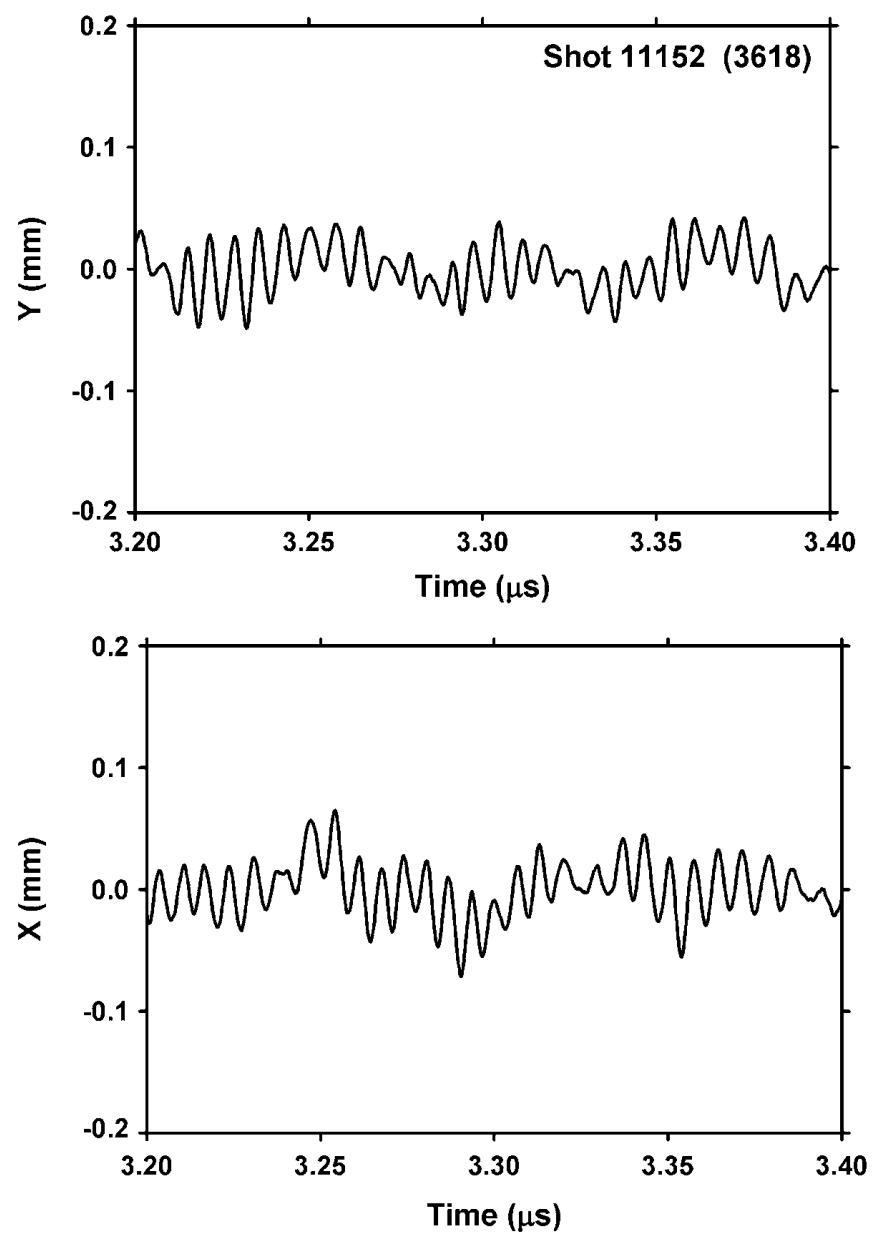

FIG. 3. High-frequency BBU motion measured at accelerator exit. The beam radius at this position predicted by our envelope code is $\sim 5 \mathrm{~mm}$.

focused regime. The amplitudes of the lowest BBU mode $(\sim 150 \mathrm{MHz})$ were measured with BPMs distributed throughout the accelerator (i.e., at various $N_{g}$ and $\gamma$ ), and were normalized to the measured amplitude at the accelerator entrance $\left(\xi_{0}\right)$. During these experiments $\left\langle 1 / B_{z}\right\rangle$ was varied by changing the magnetic tune, and we also varied the current $\left(I_{b}\right)$. Theoretically, a semilog plot of energyscaled amplitude against the various values of $I_{n} N_{g}\left\langle 1 / B_{z}\right\rangle$ should yield a straight line, with a slope of $Z_{\perp}$ [3]. The observed BBU in the present 68-cell configuration agrees with those earlier measurements, and with the theory, as shown in Fig. 4.

At the accelerator exit we recorded the BPM data at $5 \mathrm{Gs} / \mathrm{s}$ in order to have enough bandwidth to detect even the highest frequency BBU mode at $\sim 570 \mathrm{MHz}$. Figure 5 is the spectrum of beam motion recorded there, which shows that there is BBU activity in all of the cavity mode bands, including near $600 \mathrm{MHz}$, although the data at the higher frequencies was highly attenuated by the bandwidth limit of our BPM system. The measured system attenuation at $570 \mathrm{MHz}$ is $15.7 \mathrm{~dB}$ greater than the attenuation at

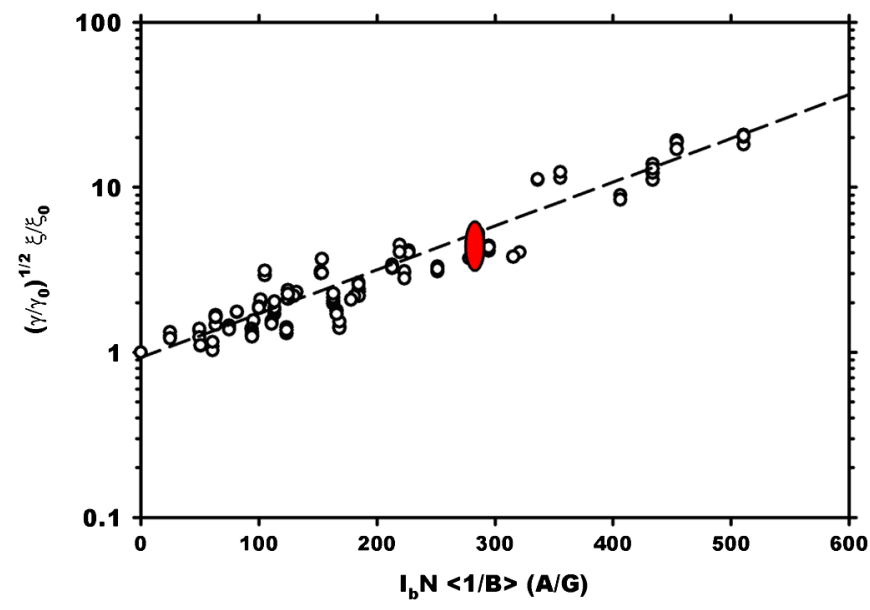

FIG. 4. Measured BBU growth. Open circles: saturated amplitude data obtained in 1.3-kA experiments with an early 50-cell configuration of the LIA [3]. Filled oval: range of saturated amplitude data obtained during 1.8-kA experiments with the present 68-cell configuration. Dashed line: best fit to data, obtained with $Z_{\perp}=184 \Omega$, compared with $157-\Omega$ average measured over the frequency band.

$150 \mathrm{MHz}$ (a factor of 6.1). Applying this factor to the peak at $\sim 570 \mathrm{MHz}$ in Fig. 5 gives an amplitude of $2.8 \mathrm{~V} / \mathrm{Hz}$, which is less than the peak at $\sim 150 \mathrm{MHz}$, implying that the highest frequency BBU displacements are no larger than the lower frequency displacements, and is also less than $2 \%$ of the beam radius. Since the theoretical gain at $570 \mathrm{MHz}$ is $\sim 7$ times that at $150 \mathrm{MHz}$ (Fig. 4), this also implies that the initial seed amplitude at the highest frequencies is much less than at low frequencies.

In addition to the observed BBU spectrum in Fig. 4, there is beam motion at $100 \mathrm{MHz}$ and below. We observe this lower frequency motion on all BPMs, including at the

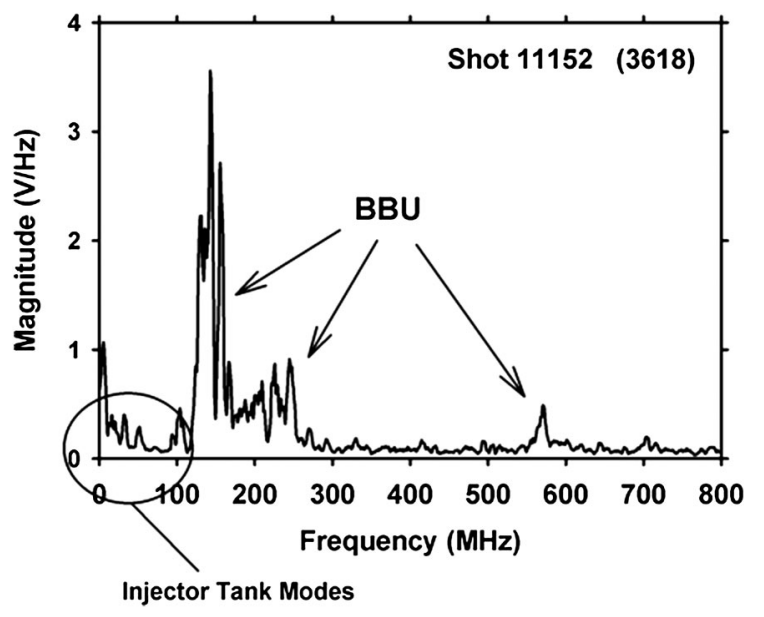

FIG. 5. Spectrum of BBU activity at $120 \mathrm{MHz}$ and above. The three predicted resonance bands that were seen in measurements of transverse impedance $[13,14]$ are clearly evident in this spectrum, as are the rf modes of the injector vacuum vessel, which were also predicted [9]. 
accelerator entrance. We attribute this motion to be the result of rf modes predicted for the large injector vacuum vessel, because the frequency peaks are in agreement with the predictions [9].

\section{B. Suppression of low-frequency beam sweep}

Low-frequency beam motion, with a period greater than the kicked pulse FWHM, would result in displacement of the centers of successive radiographic source spots. Each pulse has enough energy density to erode the converter target, so displacement of early pulses in a sequence could lead to azimuthally asymmetric target material and distorted spots for later pulses. Uncorrected beam motion at the exit of the Axis-II LIA was dominated by an energydependent sweep, with $>5 \mathrm{~mm}$ amplitude over the 1.6- $\mu \mathrm{s}$ flattop. Since this would result in wandering of the radiographic source spots by more than their size, it had to be corrected.

One major source of sweep is the folded current path in the injector. As shown in Fig. 6 the diode beam source is at right angles to the coaxial current feed from the Marx generator [1-3]. This asymmetry, and the resulting current path (Fig. 7), produces a weak transverse field in the diode anode-cathode gap that deflects the beam upward [9]. The beam deflection in the asymmetric diode and its consequences are unique to the DARHT Axis-II accelerator; all other LIAs have axisymmetric diode geometries. Because of the diode asymmetry, the beam enters the solenoidal magnetic focusing field with an upward tilt, which causes

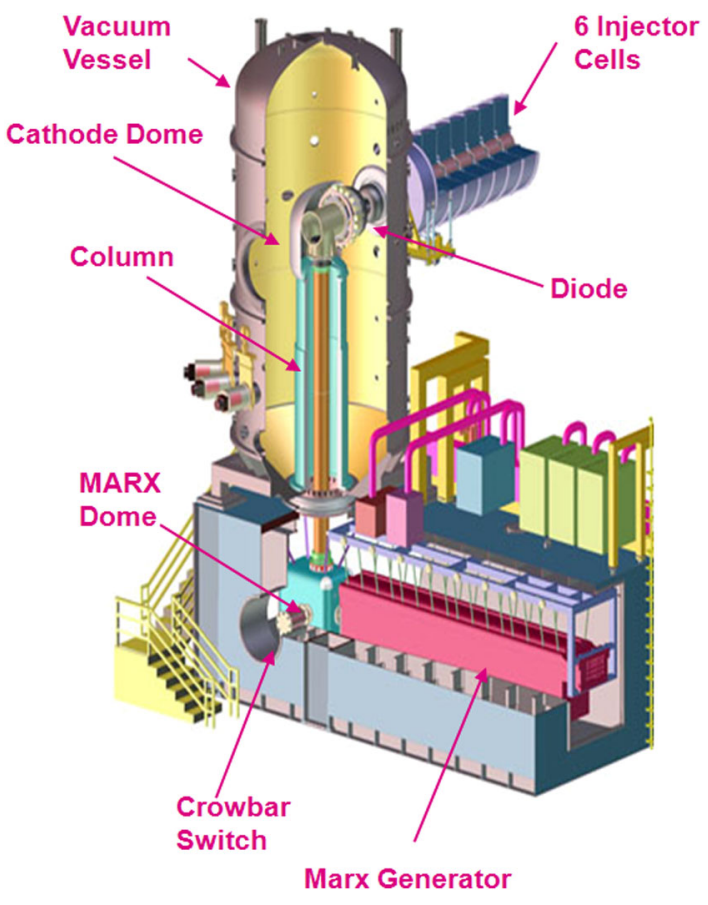

FIG. 6. Injector diagram. The (negative) current path is twice bent at right angles as it flows from the Marx generator, up the column, across the diode gap, and into the injector cells.

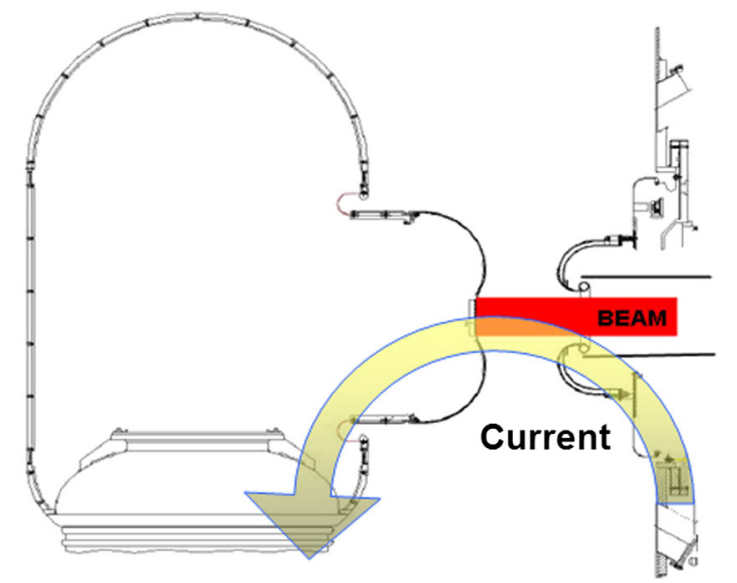

FIG. 7. Side view of source dome and diode indicating the net current path that leads to a transverse field in the diode and upward deflection of the beam.

the beam to follow a helical trajectory. This helix is initially large, and if uncorrected it remains large through the accelerator, as shown by simulations of the beam-centroid position in Figs. 8 and 9. These simulations use measured beam-centroid positions for initial conditions, magnetic field models fit to measurements for solenoidal and dipole fields, and measured solenoid misalignments. The predicted helical trajectory is stationary only if the beam initial energy and the cell accelerating potentials are constant in time. If either of these vary in time, the helix phase and gyroradius also vary at the LIA exit, causing the beamcentroid position to sweep in time, as illustrated in Fig. 10, which shows the sweep resulting from coherent variation of the accelerating-cell potentials.

Obviously, the first step toward reducing beam motion at the accelerator exit would be to minimize the size of the

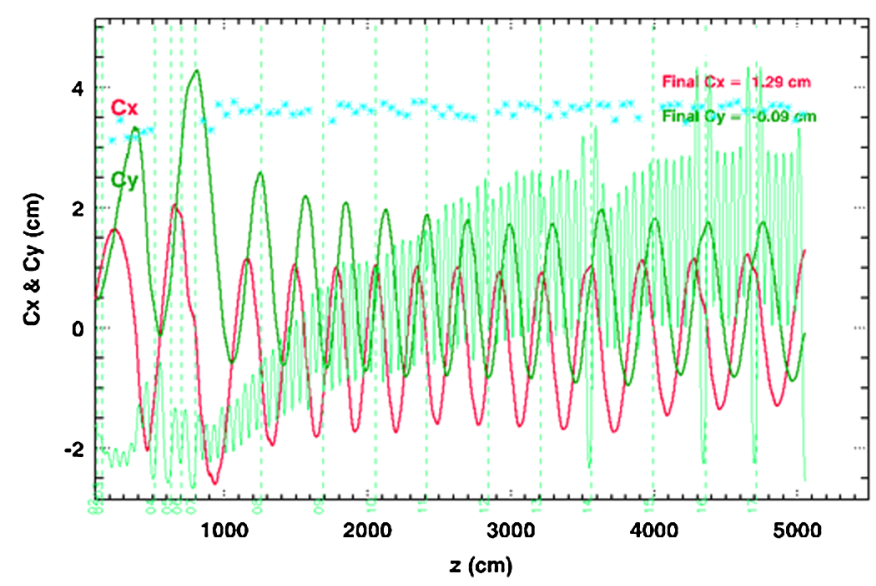

FIG. 8. Simulation of the beam-centroid position in $x$ ( $\mathrm{Cx}$ in red) and $y$ (Cy in green). For this simulation the initial offset was uncorrected, and the resulting large helical trajectory extended through the accelerator. In this simulation the helical trajectory is stationary in time, because there is no time variation of the initial energy or accelerating potentials. 


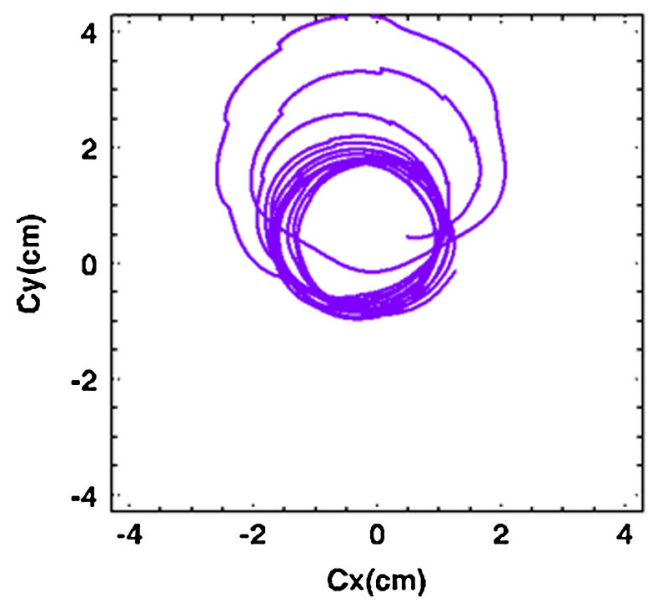

FIG. 9. End view of the helical trajectory of the simulation shown in Fig. 8. This trajectory is stationary in time if the diode and accelerating potentials are constant.

helical trajectory caused by offset injection. Therefore, we now correct the offset injection by centering the beam using dipoles in the six injector cells. Figures 11 and 12 illustrate how well this works in a simulation using only two pairs of dipoles to correct the helical trajectory shown in Figs. 8 and 9.

As seen in Fig. 11, the injector cell dipoles are very effective at centering the beam and "unwinding" the helical trajectory. However, even after correcting this initial offset effect, the trajectory again becomes helical further downstream. The source of this residual helical motion is the interaction of the beam with accidental dipoles caused

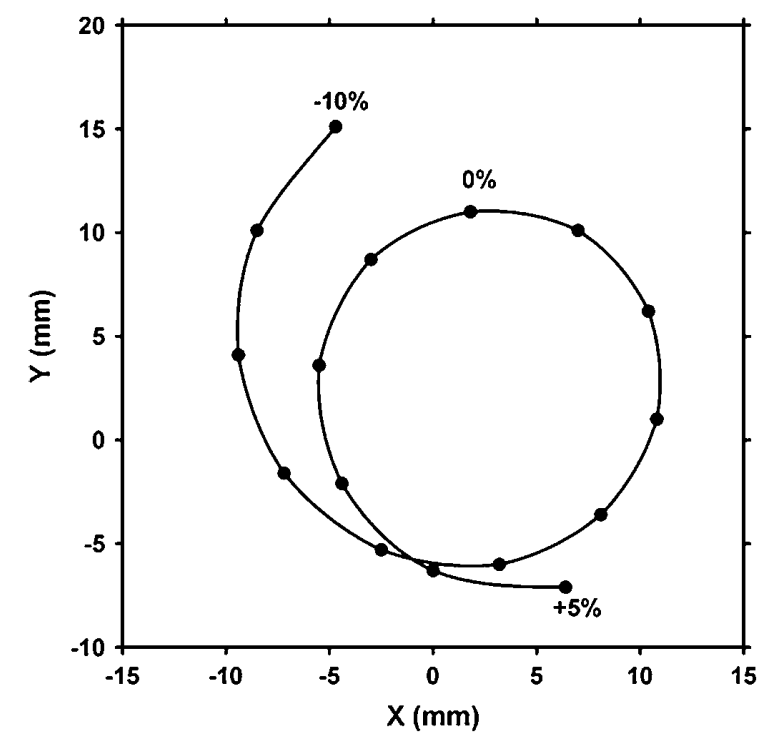

FIG. 10. Beam position at the accelerator exit for a number of simulations in which the cell accelerating potentials were all varied over a range of $15 \%$ in $1 \%$ increments (solid dots). This simulates a plot of beam-centroid position motion caused by accelerating potentials that coherently vary in time, as in the DARHT Axis-II LIA.

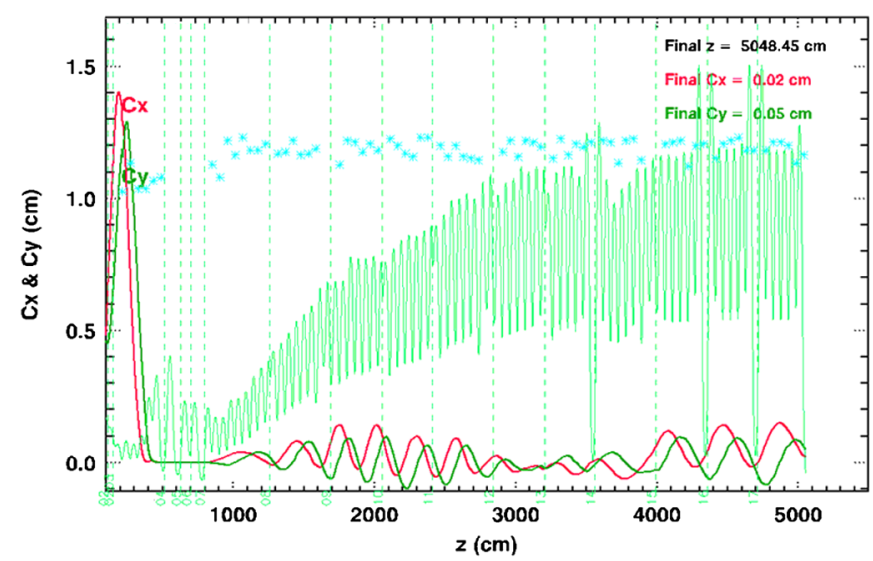

FIG. 11. Simulation of the beam-centroid position in $x(\mathrm{Cx}$ in red) and $y$ (Cy in green). For this simulation the initial offset and resulting large helical motion has been corrected using two dipole pairs in the injector cells to center the beam. The residual helical motion is the result of cell misalignments.

by small cell misalignments. This simulation modeled these dipoles based on actual measurements of the small cell misalignments; $\sim 0.1-\mathrm{mm}$ rms offset, and $\sim 0.3-\mathrm{mr}$ rms tilt.

We measure the beam kinetic energy on every shot using diode and cell voltage monitors that are cross calibrated to a magnetic spectrometer that is calibrated to better than $0.5 \%$. Figure 13 shows the measured kinetic energy variation during a 1.4- $\mu \mathrm{s}$ portion of the flattop of the pulse during a single shot $(\sim 2.5 \%)$. (All of the beam position measurements reported here are over that same portion of the flattop.) Figures 14 and 15 show the motion of the beam-centroid position at the accelerator exit due to the energy variation shown in Fig. 13. The strong correlation of beam sweep with energy variation is clearly seen by comparing Fig. 15 with Fig. 13. The total range of motion

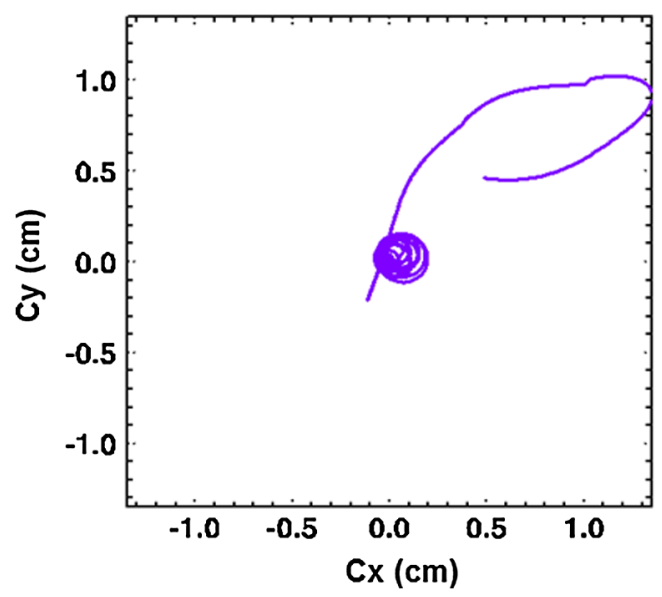

FIG. 12. End view of the helical trajectory of the simulation shown in Fig. 11 for a beam that is initially centered and the trajectory straightened at the exit of the injector cells. (Compare with Fig. 9 for an uncentered beam.) 


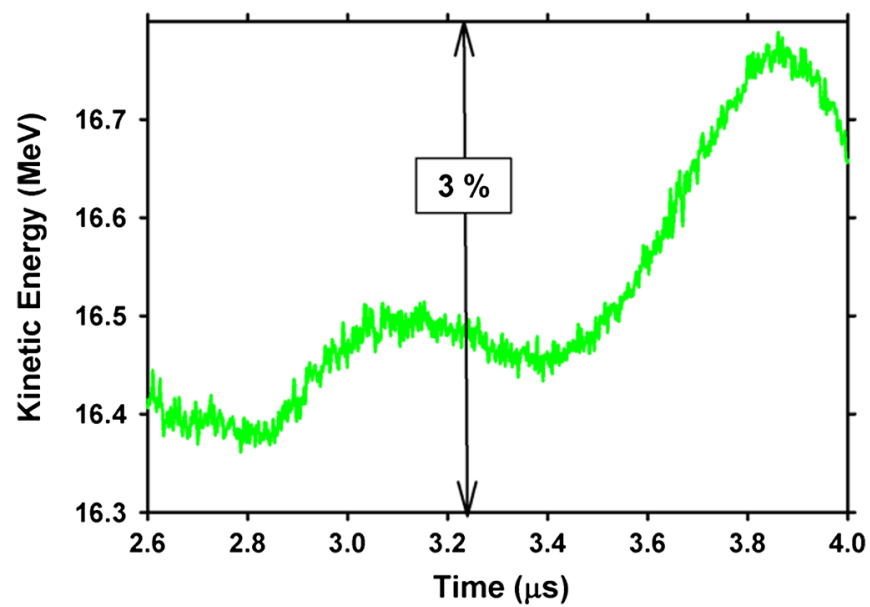

FIG. 13. Beam kinetic energy at exit of the LIA measured by summing voltage monitors that have been cross calibrated to accurate magnetic spectrometer measurements of beam kinetic energy. The fractional beam energy variation at any point in the LIA closely resembles this, because the oscillation is a characteristic of the pulsed-power accelerating-cell drivers.

encompasses $\sim 110^{\circ}$ of a fitted arc, which is in agreement with the range predicted by the simulations for a comparable energy variation (Fig. 10), thereby substantially validating the simulations.

The beam kinetic energy variation shown in Fig. 13 is characterized by a $\sim 1.5 \mathrm{MHz}$ oscillation and a monotone increase. This waveform is typical of the individual cell pulsed-power drivers, and so the kinetic energy variation is

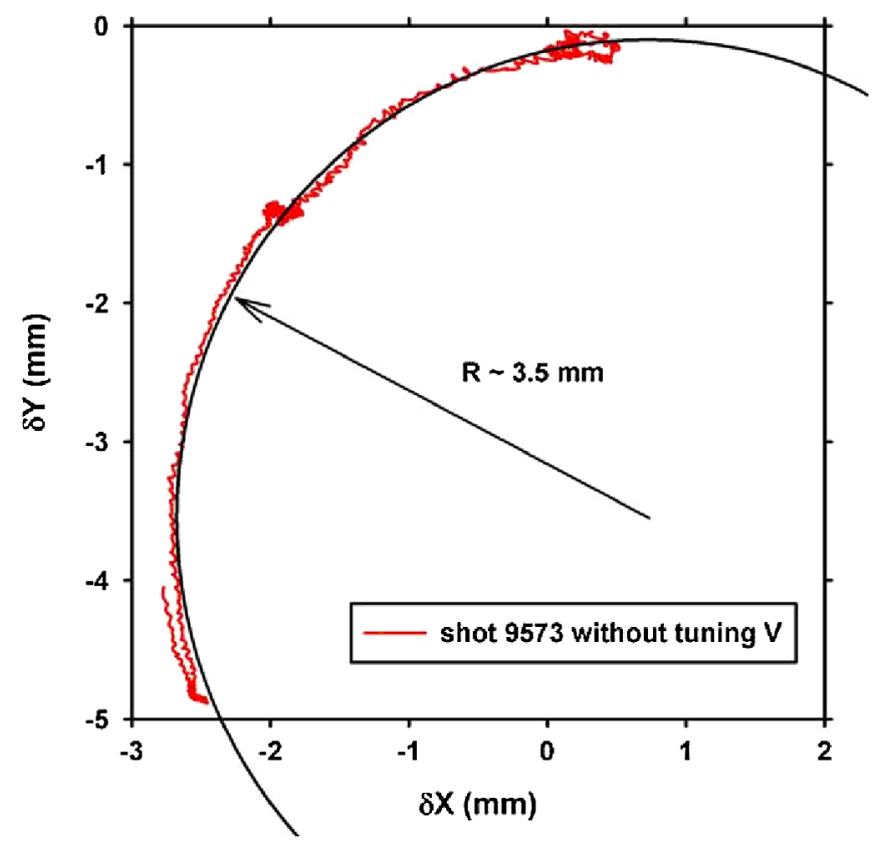

FIG. 14. Motion of beam-centroid position measured with the BPM at the LIA exit. This motion was caused by the energy variation shown in Fig. 13 (measured on the same shot). The angular motion is in the counterclockwise (ccw) direction.

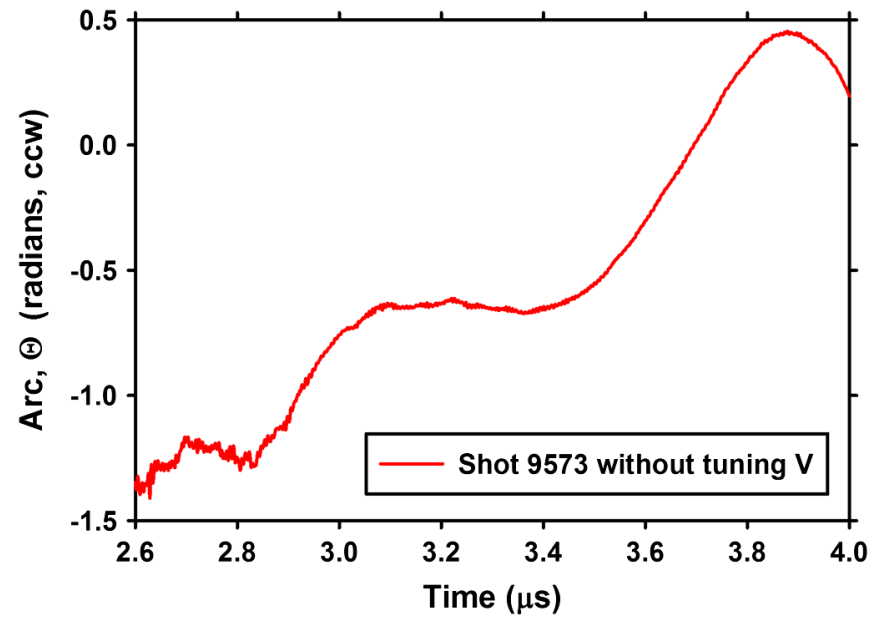

FIG. 15. Angular motion of beam centroid shown in Fig. 14 plotted vs time to emphasize the correlation with the energy variation shown in Fig. 13.

coherent throughout the LIA. That is, the fractional beam energy variation at any point closely resembles Fig. 13. As a consequence, the resulting helical phase advance due to deflection by misalignment dipoles accumulates. Indeed, the observed phase angle sweep at the exit can be roughly fit with a model of deflection by a long transverse magnetic field, as shown in Fig. 16. The model simply assumes deflection inversely proportional to the beam momentum, as if by a transverse (dipole) field.

The strong correlation of sweep with beam energy strengthens the argument that the cause of the sweep is the energy-dependent interaction with misalignment dipoles. Moreover, this correlation argues against an instability causing the sweep, unless the instability has strong

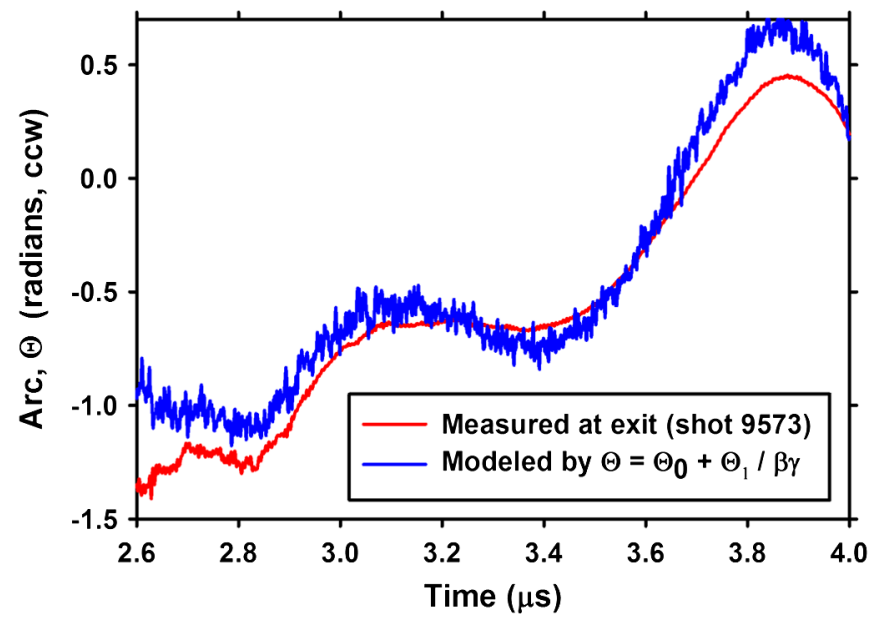

FIG. 16. Uncorrected beam position on the helical surface at LIA exit. (Red): The red curve is the phase angle of the position measurements shown in Figs. 14 and 15. (Blue): The blue curve is the phase angle modeled as if a beam with the measured kinetic energy variation shown in Fig. 13 were deflected by a single dipole. 
energy dependence. For example, head-to-tail sweep is a characteristic of the resistive wall instability [15]. In a uniform strong solenoidal focusing field, the distance for an initial perturbation to exponentiate due to the resistive wall effect is approximately $L=3.1 B a^{3} / I_{b} /(\tau \rho)^{1 / 2}$ :

$$
L \approx 3.1 \frac{B_{z} a^{3}}{I_{b \sqrt{\rho \tau}}}
$$

where $a$ is the pipe radius in $\mathrm{cm}, B_{z}$ is the solenoidal field in $\mathrm{kG}, I_{b}$ is the beam current in $\mathrm{kA}, \tau$ is the pulse length in $\mu \mathrm{s}$, and $\rho$ is the pipe resistivity in $\mu \Omega-\mathrm{cm}[15,16]$. The inverse root scaling with pulse length, and the inverse scaling with current, imply that this instability could be problematic for long-pulse, high-current accelerators, like the Axis-II LIA. However, the resistive wall growth is independent of energy, in contradiction to our sweep data. Moreover, we estimate that the growth of an initial perturbation in DARHT-II is less than $60 \%$ over the length of the LIA, largely because of the large bore $(25-\mathrm{cm}$ diameter) beam pipe. Therefore, it is unlikely that the sweep is caused by resistive wall instability.

Beam energy variations coupling with focusing magnet misalignments is the source of the "corkscrew" motion [17] observed in the ETA-II LIA [18-21]. Moreover, suppression of this energy-dependent motion by using steering dipoles throughout the LIA in a procedure known as a "tuning V" has been demonstrated [18-21]. However, the procedures employed on ETA-II used between 12 and

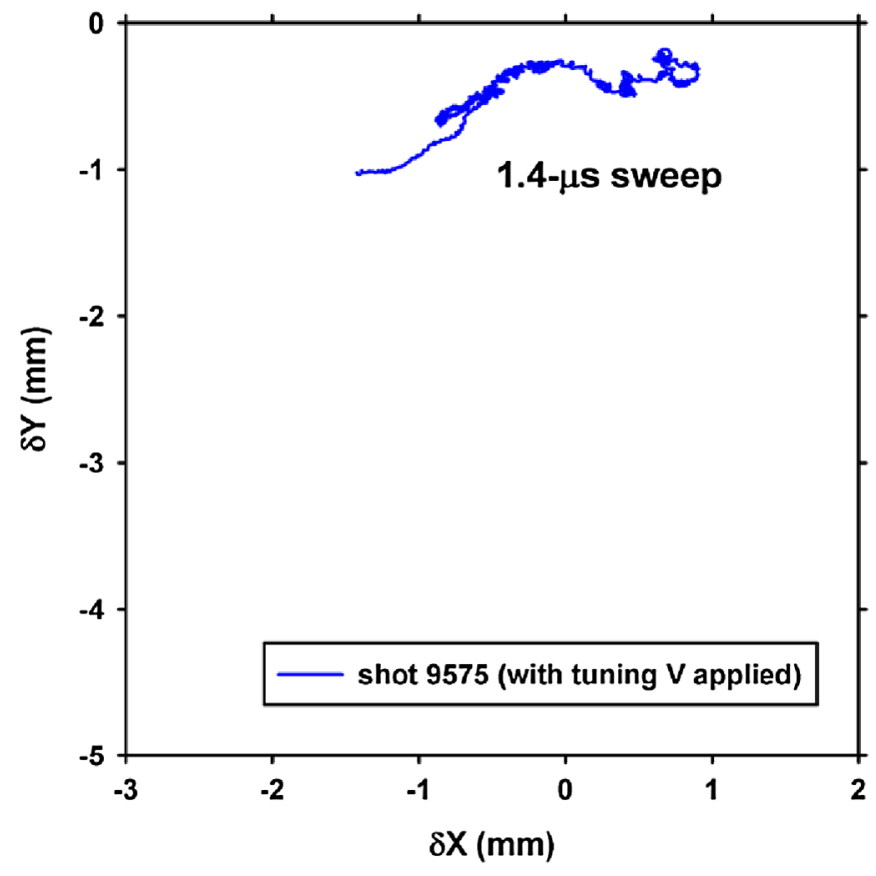

FIG. 17. Reduction of sweep through the use of two pairs of corrector dipoles following the "tuning-V" procedure [16,17]. (Compare with Fig. 14.)

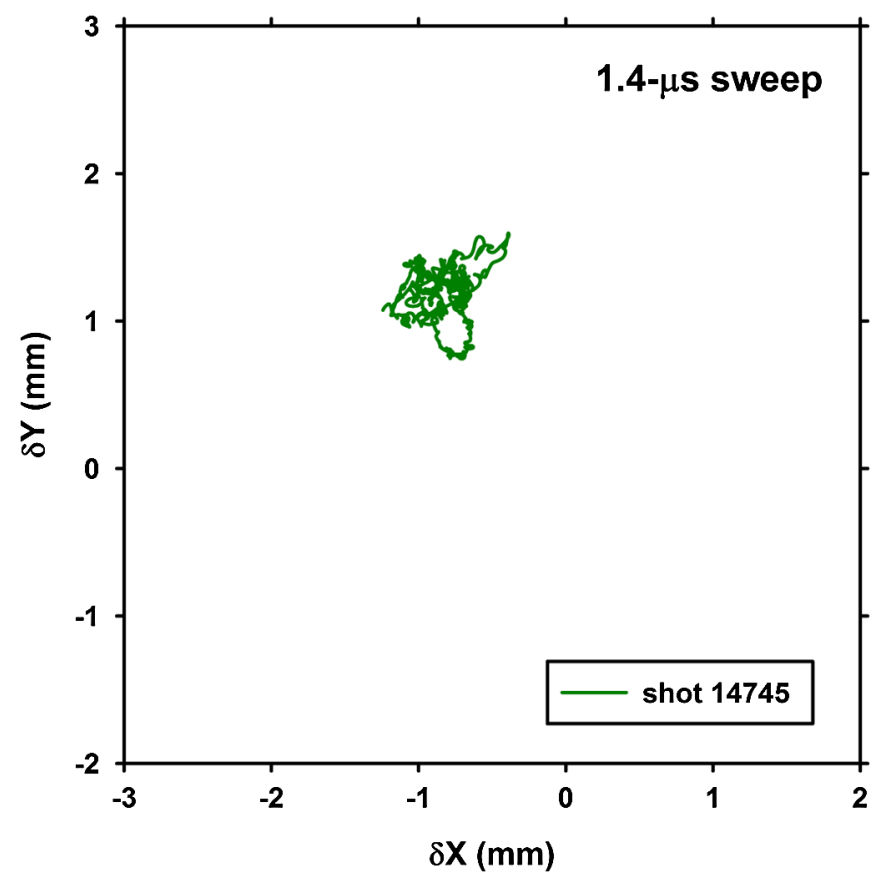

FIG. 18. Further reduction of sweep obtained by first centering the beam and unwinding the helical trajectory at the injector, and then applying tuning Vs at two different locations. (Compare with Fig. 17.)

60 dipole pairs, and required between one hour and one day at the 1-pulse/s repetition rate of ETA-II [19-21]. Clearly, that would be impractical on our accelerator, because we seldom achieve more than 40 beam shots per day, so we have developed a new tuning- $\mathrm{V}$ procedure requiring far fewer dipoles and shots.

Only a few appropriately applied corrector dipole are needed to correct the sweep, even though the corrector fields cannot exactly cancel the misalignment fields because they are not colocated. Using only two of the 68 available pairs of dipoles in the main accelerator, we were able to significantly reduce the sweep amplitude (Fig. 17) with our new tuning-V procedure. This initial attempt reduced the sweep amplitude by $>50 \%$ from $>5$ to $<2.5 \mathrm{~mm}$, with the amplitude defined as the diagonal of the bounding rectangle. Since then, we have improved on that by more accurate centering and unwinding the helical trajectory of the beam out of the injector (like the simulation shown in Figs. 11 and 12). Again using only two dipole pairs for tuning Vs, the sweep was further reduced by another factor of 2 to $\sim 1.2 \mathrm{~mm}$ (Fig. 18) in only 21 beam shots. This is $<25 \%$ of the $\sim 5$-mm envelope radius predicted at the BPM location just outside of the accelerator. The four radiograph pulses are spaced over a somewhat longer time span $(1.53 \mu \mathrm{s})$, so the sweep at these pulse times is slightly larger $(\sim 1.5 \mathrm{~mm})$, but still less than $30 \%$ of the predicted beam envelope radius. This suggests that the spot-to-spot wander of the focused beam may be less than $15 \%$ of the radiographic source spot FWHM. 


\section{DISCUSSIONS AND REMARKS}

We have suppressed beam motion in the Axis-II LIA to amplitudes small enough to have little effect on radiographic performance. The solenoidal magnetic focusing field was strong enough to suppress the BBU to less $\sim 2 \%$ of the beam radius, so it will have little effect on source spot size. Low-frequency beam sweep was reduced to less than $1 / 3$ of the beam radius, so the resulting displacement of source spots should be less than $20 \%$ of the spot FWHM. The kinetic energy waveform is the cumulative sum of the waveforms typical of the cell pulsed-power drivers, so it can be flattened somewhat by randomizing the cell timing within limits prescribed by beam transport considerations. Future efforts to further reduce the sweep include this approach to minimize the kinetic energy variation, which is the source of the problem.

\section{ACKNOWLEDGMENTS}

This work was supported by the U.S. National Nuclear Security Agency and the U.S. Department of Energy under Contract No. W-7405-ENG-36.

[1] Carl Ekdahl et al., in Proceedings of the 20th Particle Accelerator Conference, Portland, OR, 2003 (IEEE, New York, 2003), p. 558.

[2] Carl Ekdahl et al., IEEE Trans. Plasma Sci. 33, 892 (2005).

[3] Carl Ekdahl et al., IEEE Trans. Plasma Sci. 34, 460 (2006).

[4] Martin Schulze et al., in Proceedings of the XXIV Linear Accelerator Conference, Victoria, Canada, 2008, p. 427 [http://accelconf.web.cern.ch/accelconf//LINAC08/papers/ tup020.pdf].

[5] Thomas P. Hughes, David C. Moir, and Paul W. Allison, in Proceedings of the Particle Accelerator Conference, Dallas, TX, 1995 (IEEE, New York, 1995), p. 1207.
[6] Thomas P. Hughes et al., Voss Scientific Technical Report No. VSL-0707, 2007.

[7] E.P. Lee and R. K. Cooper, Part. Accel. 7, 83 (1976).

[8] Stanley Humphries, Jr., in Computational Accelerator Physics, edited by R. Ryne (American Institute of Physics, New York, 1994), p. 587.

[9] T. P. Hughes, R. E. Clark, and S. S. Yu, Phys. Rev. ST Accel. Beams 2, 110401 (1999).

[10] Carl Ekdahl, Rev. Sci. Instrum. 76, 095108 (2005).

[11] H. Bender et al., Rev. Sci. Instrum. 78, 013301 (2007).

[12] V. K. Neil, L. S. Hall, and R. K. Cooper, Part. Accel. 9, 213 (1979).

[13] R. Briggs et al., in Proceedings of the Particle Accelerator Conference, Chicago, IL, 2001 (IEEE, New York, 2001), p. 1850.

[14] H.L. Rutkowski et al., in Proceedings of the XX Linear Accelerator Conference, Monterey, CA, 2000, p. 449 [http://accelconf.web.cern.ch/accelconf/100/papers/TUB08 .pdf].

[15] G. J. Caporaso, W. A. Barletta, and V. K. Neil, Part. Accel. 11, 71 (1980).

[16] Carl Ekdahl et al., in Proceedings of the 23rd Particle Accelerator Conference, Vancouver, Canada, 2009 (IEEE, Piscataway, NJ, 2009), p. 3080.

[17] Y.-J. Chen, Nucl. Instrum. Methods Phys. Res., Sect. A 292, 455 (1990).

[18] Y.-J. Chen, Nucl. Instrum. Methods Phys. Res., Sect. A 398, 139 (1997).

[19] S.L. Allen et al, in Proceedings of the 1991 Particle Accelerator Conference, San Francisco, CA, 1991 (IEEE, New York, 1991), p. 3094.

[20] S. L. Allen and E. T. Scharlemann, in Proceedings of the 9th International Conference on High Power Particle Beams Washington, DC, 1992, p. 247 [National Technical Information Service (NTIS) Report No. PB92206168].

[21] J. T. Weir et al., in Proceedings of the 1999 Particle Accelerator Conference, New York, NY, 1999 (IEEE, New York, 1999), p. 3513. 